\title{
SEPTEMBRE 1957
}

\section{REVUE}

\section{INTERNATIONA LE}

DE LA

\section{CROIX-ROUGE}

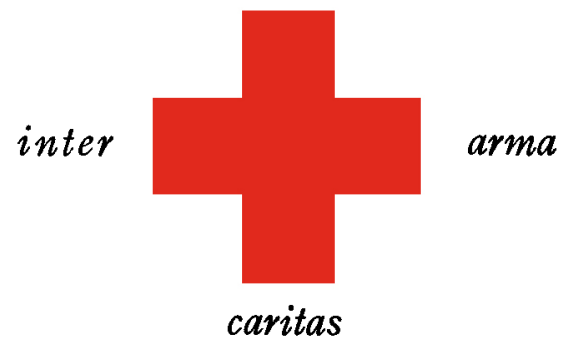

COMITÉ INTERNATIONAL DE LA CROIX-ROUGE G E N È E 


\section{COMITÉ INTERNATIONAL DE LA CROIX-ROUGE à Genève}

MM. MAX HUBER, docteur en droit, ancien président de la Cour permanente de justice intemationale président d'honneur (1923) 1

LÉOPOLD BOISSIER, docteur en droit, professeur honoraire de l'Université de Genève, ancien secrétaire général de 1'Union interparlementaire, président (1946)

- JACQUES CHENEVIERE, docteur ès lettres h. c. (1919)

Mi1e LUCIE ODIER, ex-chef du Service des infirmières-visiteuses de la Section genevoise de la CroixRouge suisse (1930)

MM. CARL J. BURCKHARDT, docteur en philosophie, ancien ministre de Suisse en France (1933)

MARTIN BODMER, docteur en philosophie b. c., vice-président (1940)

ERNEST GLOOR, docteur en médecine (1945)

PAUL CARRY, docteur en droit, professeur à l'Université de Genève (1946)

EDMOND GRASSET, docteur en médecine, professeur à l'Université de Genève, directeır de l'Institut d'hygiène (1946)

PAUL RUEGGER, ancien ministre de Suisse en Italie et en Grande-Bretagne, membre cle la Cour Permanente d'Arbitrage (1948)

HENRI GUISAN, général, ancien commandant en chef de l'armée suisse (1948)

ALFREDO VANNOTTI, docteur en médecine, professeur à l'Université de Lausanne (1949)

RODOLFO OLGIATI, ancien directeur du Don suisse (1949)

M11 MARGUERITE VAN BERCHEM, ancien chef de service de l'Agence centrale des prisonniers de guerre (1951)

MM. FRÉDÉRIC SIORDET, avocat, conseiller du Comité international de la Croix-Rouge de 1943 à 1951, vice-président (1951)

MARCEL JUNOD, docteur en médecine, délégué du Comité international de la Croix-Rouge de 1935 à 1946 (1952)

GUILLAUME BORDIER, ingénieur dipl. E.P.F., M.B.A. Harvard, banquier (1955)

\section{Direction:}

MM. ROGER GALLOPIN, docteur en droit, directeur exécutif

JEAN S. PICTET, docteur en droit, directeur des Affaires générales

EDOUARD de BONDELI, sous-directeur, Services financiers et administratifs

CLAUDE PILLOUD, sous-directeur, Service juridique

1 Les années indiquees dans les parenthèses désignent les dates de nomination des membres du Comité international. 


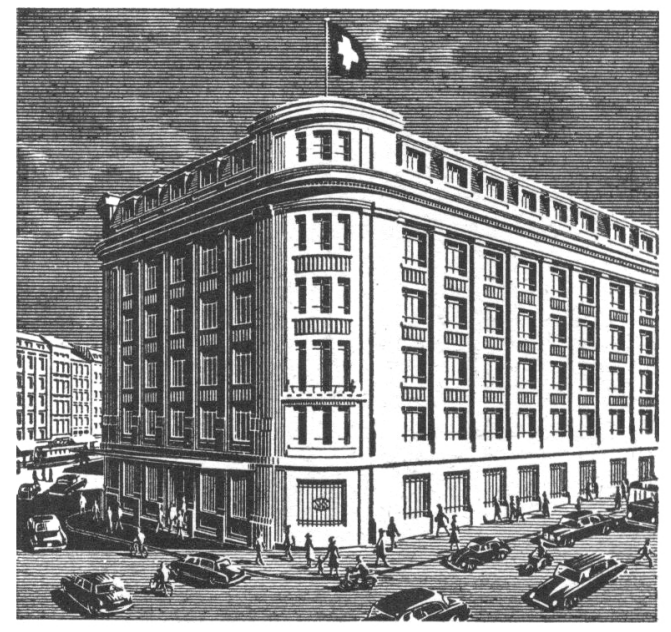

\section{SOCIETÉ DE}

\section{BANQUE SUISSE} SCHWEIZERISCHER BANKVEREIN SOCIETÀ DI BANCA SVIZZERA SWISS BANK CORPORATION

\section{GEN ÈVE}

BALE BIENNE LA CHAUX-DE-FONDS LAUSANNE NEUCHATEL ST-GALL SCHAFFHOUSE ZURICH LONDRES NEW-YORK

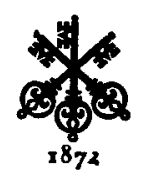

CAPITAL ET RÉSERVES FRS 275 MILLIONS 


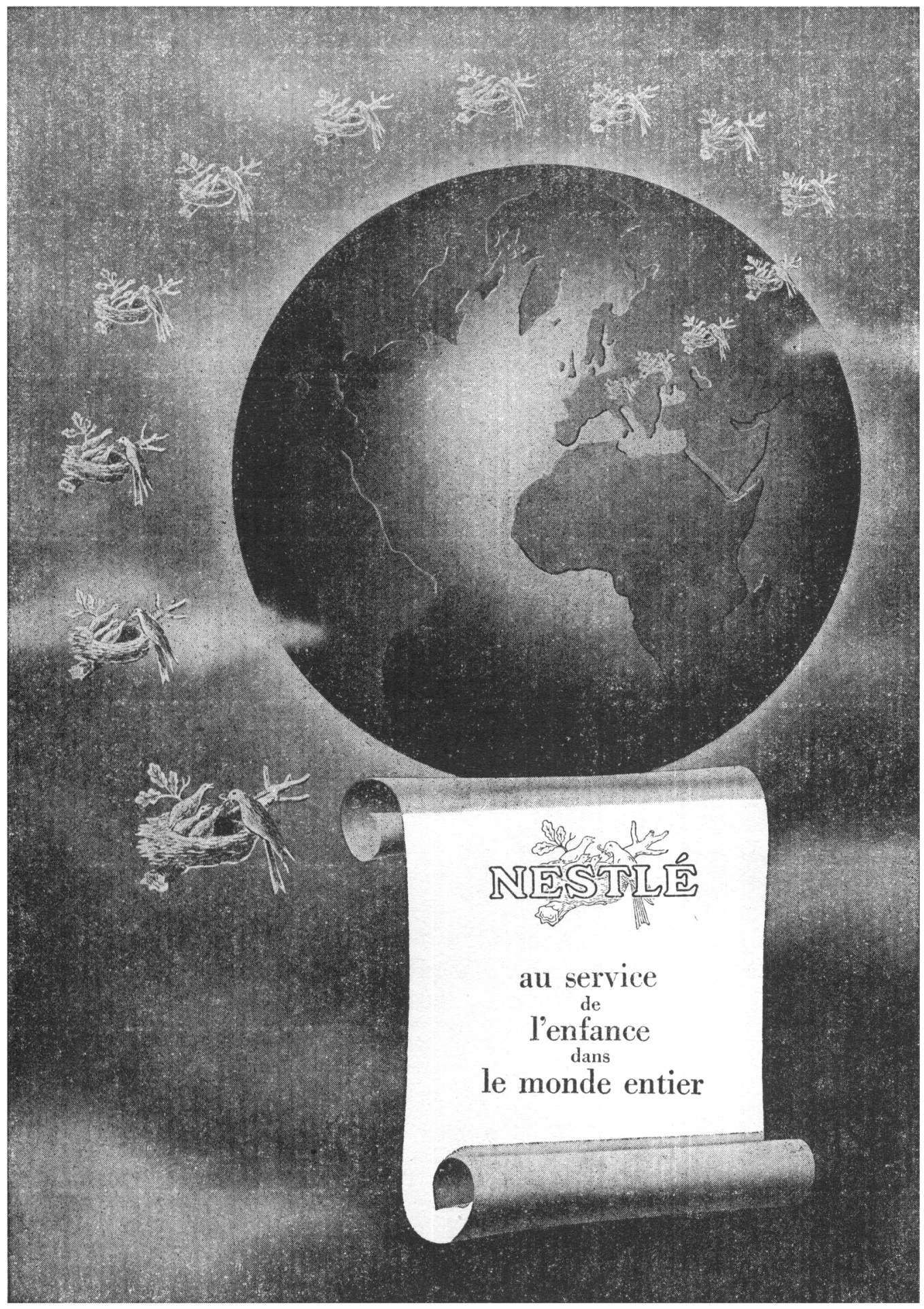




\title{
REVUE INTERNATIONALE \\ DE LA CROIX-ROUGE
}

\author{
TRENTE-NEUVIÈME ANNEE - No 465 \\ SEPTEMBRE 1957
}

\section{SOMMAIRE}

Pages

COMITE INTERNATIONAL DE LA CROIX-ROUGE

Assistance traditionnelle aux victimes des conflits et de leurs suites $\quad 47 \mathrm{r}$

La protection des médecins civils en temps de conflit (J.-P.S.) 485

Emissions de la Croix-Rouge en langue arabe . . . . . . . 494

\section{CROIX-ROUGE INTERNATIONALE}

La XIXe Conférence internationale de la Croix-Rouge va s'ouvrir (J. P. et C. P.) . . . . . . . . . . . . . . .

\section{NOUVELLES DE SOCIÉTÉS NATIONALES}

Corée (République démocratique) . . . . . . . . . . . 504 Israël . . . . . . . . . . . . . . . . . . . . . 505

Pays-Bas . . . . . . . . . . . . . . . . . . . . 507

\section{CHRONIQUE}

Infirmières et assistantes sociales d'une Croix-Rouge nationale

(J.G. L.) . . . . . . . . . . . . . . . . . 5 II

FAITS ET DOCUMENTS

Problèmes de la santé publique . . . . . . . . . . . . . $52 \mathrm{I}$

\section{A TRAVERS LES REVUES}




\section{SUPPLÉMENTS DE LA REVUE}

EN LANGUE ANGLAISE

Traditional Forms of assistance to the victims of conflicts and their after-effects. - The protection of civilian members of the medical professions in time of armed conflict (J.-P. S.).

EN LANGUE ESPAGNOLE

Asistencia tradicional a las víctimas de los conflictos y de sus consecuencias. - La protección de los médicos civiles en tiempo de conflicto (J.-P. S.).

EN LANGUE ALLEMANDE

Der traditionelle Beistand an die Opfer von Konflikten und deren Folgen. - Der Schutz der Zivilärzte in Kriegszeiten (J.-P. S.).

LA

REVUE INTERNATIONALE DE LA CROIX-ROUGE est publiée chaque mois par le Comité international de la Croix-Rouge

7, avenue de la Paix, Genève (Suisse) - Compte de chèques postaux I. 1767

Abonnement un an : Fr. 20,- ; le numéro Fr. 2,-

Pour les pays suivants : Algérie, Állemagne, Autriche, Belgique, Danemark, Finlande, France, Italie, Luxembourg, Maroc, Norvdge, Pays-Bas, Portugal, Sarre, Suede, Tunisie, Vatican, on peut s'abonner auprès des bureaux de poste (Fr. s. 20,50).

Rédaction : JEAN-G. LOSSIER 